\section{OPEN ACCESS}

Edited by Karl Kuchler,

Medical University of Vienna, Austria

Reviewed by:

Glen C. Ulett,

Griffith University, Australia

Sven Hammerschmidt,

University of Greifswald, Germany

*Correspondence:

Thomas Emil Andersen thandersen@health.sdu.dk

Lars Lund

Lars.Lund@rsyd.dk

tThese authors have contributed equally to this work

Specialty section

This article was submitted to Infectious Diseases,

a section of the journal

Frontiers in Microbiology

Received: 10 May 2019

Accepted: 23 October 2019

Published: 21 November 2019

Citation:

Nielsen TK, Petersen NA,

Stærk K, Grønnemose RB,

Palarasah Y, Nielsen LF, Kolmos HJ, Andersen TE and Lund L (2019) A

Porcine Model for Urinary Tract Infection. Front. Microbiol. 10:2564.

doi: 10.3389/fmicb.2019.02564

\title{
A Porcine Model for Urinary Tract Infection
}

\begin{abstract}
Thomas Kastberg Nielsen ${ }^{1+}$, Nicky Anúel Petersen ${ }^{1 \dagger}$, Kristian Stærk $^{2+}$, Rasmus Birkholm Grønnemose ${ }^{2}$, Yaseelan Palarasah ${ }^{3}$, Lene Feldskov Nielsen ${ }^{4}$, Hans Jørn Kolmos ${ }^{2}$, Thomas Emil Andersen ${ }^{2 *}$ and Lars Lund ${ }^{1 *}$
\end{abstract}

\begin{abstract}
${ }^{1}$ Research Unit of Urology, Department of Clinical Research, Odense University Hospital, University of Southern Denmark, Odense, Denmark, ${ }^{2}$ Research Unit of Clinical Microbiology, Department of Clinical Research, Odense University Hospital, University of Southern Denmark, Odense, Denmark, ${ }^{3}$ Department of Cancer and Inflammation, University of Southern Denmark, Odense, Denmark, ${ }^{4}$ Coloplast A/S, Humlebaek, Denmark
\end{abstract}

Urinary tract infection (UTI) is the most common bacterial infectious disease with a high frequency of recurrence and the leading cause of septicemia. In vivo experimentation has contributed significantly to the present-day knowledge on UTI pathogenesis. This research has traditionally been based on murine models of UTI. Occasional conflicting results between UTI in mice and humans and increasing skepticism toward small rodent models in general warrant the need of novel large-animal infection models that better resemble the anatomy and physiology of humans, and thus better mimic the course of infection in humans. Here, we report, to our knowledge, the first large-animal model of cystitis. The model is based on pigs, and the protocol supports the establishment of persistent, non-ascending infection in this animal and is established without invasive surgical procedures, pain, and discomfort for the animal. The course of infection is monitored by cystoscopy, microscopy of bladder biopsies, and biochemical analysis of urine and blood samples. At termination, harvested whole bladders from infected pigs are analyzed for microbiological colonization using microscopy, histology, and viable bacterial counts. The model is a useful tool in future studies of UTI pathogenesis and opens up novel possibilities to bridge the current knowledge obtained from small-animal UTI models to UTI pathogenesis in humans.

Keywords: cystitis, porcine model, urinary tract infection, Escherichia coli, UPEC, swine

\section{INTRODUCTION}

Urinary tract infections (UTIs) are the most common bacterial infection with an annual incidence of community-acquired UTIs of more than $10 \%$ for women in the United States (Foxman, 2014). This high incidence is partly explained by the prevalent frequency of recurrent infections following approximately $25-40 \%$ of acute UTIs and bringing about severe morbidity in otherwise healthy women (Foxmann, 2010). In Western countries, UTIs also constitute the major part of hospitalacquired infection accounting for 13-19\% of cases (European Center for Disease Control, and Prevention [ECDC], 2013; Magill et al., 2014) with a significant risk of progression to urosepsis as is seen in up to $25 \%$ of patients (Bjerklund Johansen et al., 2007; Wagenlehner et al., 2008; Tandoğdu et al., 2016). 
Treating and preventing UTI is challenging, and the problem is unlikely to diminish in the future. Improved hygiene procedures and antibiotic stewardship are important steps to control UTIs (Cai et al., 2016). However, a better fundamental understanding of UTI pathogenesis is critical to improve treatment regimens and develop more targeted antimicrobials.

Uropathogenic Escherichia coli (UPEC) is by far the most frequent uropathogen in both hospitalized patients and in the general population (Foxmann, 2010; Tandogdu et al., 2014). This bacterium and its role in UTI have been investigated extensively over the past decades. Murine models of UTI have been the main tool to elucidate $E$. coli infection pathogenesis (Hung et al., 2009; Carey et al., 2016). Although these studies have provided important knowledge regarding UTI pathogenesis, critical pathogenic mechanisms described in mice are not always consistent with observations done in humans (Hagan et al., 2010; Bielecki et al., 2014; Subashchandrabose et al., 2014; Carey et al., 2016; Stærk et al., 2016). A growing skepticism toward murine models as predictors of human disease in general, further emphasizes the need for large animal models that more accurately reflect pathogenesis and therapeutic effects in humans (Meurens et al., 2012; Perrin, 2014).

Emerging evidence suggests that the immune system proteins in humans are closer related to pig homologs than to mice homologs (Meurens et al., 2012; Seok et al., 2013; Barber, 2016; Dawson et al., 2017). This supports using pigs as intermediate species between rodents and humans to model infection pathogenesis and immune function relevant to humans. The size and anatomical resemblance of pigs and humans also support the use of urological medical equipment such as cystoscopes and urinary catheters, allowing detailed monitoring of the progression of infection and studies of device-associated UTI. The purpose of the current study was to establish a large animal model of cystitis to facilitate the investigation of UPEC pathogenesis and novel therapeutic strategies. We based the infection protocol on a widely used murine UTI model that has been adopted in our laboratory as well (Hung et al., 2009), since this would render possible direct comparison of infection pathogenesis in the two animals in future studies.

A critical step for UPEC to successfully colonize the bladder is the ability to adhere to the epithelial mucosa lining the luminal surface of the urinary tract (Guglietta, 2017). In vitro infection experiments with human urothelial cell cultures shows that UPEC establishes as a sessile population on the surface of the epithelium that depends on and leads to upregulation of adhesive fimbriae such as type-1 fimbriae (Stærk et al., 2016; Pedersen et al., 2018). However, bacterial epithelial association and possible biofilm formation during infection in vivo is poorly described and has to our knowledge never been studied in humans or largeanimal models. This is despite that biofilm production may be a key determinant for the persistence of UPEC in the urinary tract (Soto et al., 2006). Using the porcine model, we inspected the distribution and quantified urothelial-associated bacteria during the course of infection in vivo.

The protocol presented supports establishment of a persistent infection in the porcine bladder for prolonged experiments up to 23 days with a continuant substantial colonization of the bladder mucosa. During the infection, the pigs muster a robust inflammatory response. Although the porcine UTI model, compared to a murine UTI model, suffers from reduced scalability, and a higher cost per animal, the model offers novel possibilities to investigate the pathogenic mechanisms and host response of UPEC bladder infection, which should help advancing our understanding of UPEC pathogenesis in humans.

\section{MATERIALS AND METHODS}

\section{Bacterial Strains and Growth Conditions}

For inoculation, we used UTI89, a human cystitis-derived UPEC isolate of serotype $\mathrm{O} 18: \mathrm{K} 1: \mathrm{H} 7$, widely used in several in vitro and in vivo infection assays (Mulvey et al., 2001; Justice et al., 2006; Khandige et al., 2016; Stærk et al., 2016). A green fluorescent protein-expressing variant of UTI89 (hereafter UTI89pMAN01) was used in selected infections to visualize E. coli on bladder tissue samples. This strain harbors the pMAN01 plasmid containing a transcriptional fusion of the $g f p+$ gene to the hsp60 promoter, allowing for constitutive expression of the GFP+ protein (Khandige et al., 2016). Before inoculation, UTI89 was precultured to ensure optimal type-1 fimbrial expression, similarly to the murine UTI model protocol (Hung et al., 2009). In short, a single-colony of UTI89 from an overnight (ON) LB agar plate culture was used to inoculate $25 \mathrm{ml}$ of LB broth in a $50 \mathrm{ml}$ tube (Sarstedt) and incubated statically at $37^{\circ} \mathrm{C}$ overnight. The next day, $25 \mu \mathrm{l}$ of ON culture was transferred to $25 \mathrm{ml}$ of fresh LB broth and incubated statically at $37^{\circ} \mathrm{C}$ ON. Immediately before inoculation of the pigs, bacteria were pelleted by centrifugation at $2500 \mathrm{~g}$ for $20 \mathrm{~min}$, and inoculation suspensions prepared by resuspending bacteria in $0.9 \% \mathrm{NaCl}$ saline water, adjusting to an optical density of 1.00 at $600 \mathrm{~nm}$ and diluting this suspension further 1:10 to reach approximately $1 \times 10^{8}$ colony forming units $(\mathrm{CFU}) / \mathrm{ml}$.

\section{Animals Selection and Experimental Ethics}

Twelve female (Landrace/Yorkshire/Duroc) pigs weighing between 35.7 and $38.6 \mathrm{~kg}$ were used. The animal protocol established was designed to minimize animal pain and resulted in only mild to moderate degree of discomfort. The pigs were housed in the university hospital animal facility in standardized $3 \mathrm{~m}^{2}$ hay-covered pig enclosures and fed with standard feed and ad libitum tap water and were allowed to acclimatize and recover from the stress of transportation for 3-5 days preceding the experiments. Experiments were approved by the Danish Animal Experiments Inspectorate, license number: 2017-15-0201-01271.

\section{Anesthesia}

Adequate and correct anesthetization of the pigs are critical to reducing discomfort and stress during inoculation. In addition, inadequate sedation can result in the failure of relaxing the inner and outer urethral sphincters of the pig, thus complicating the catheterization and increasing the risk of injuring the inner-linings of the urethra and bladder, possibly making 
the pig more susceptible to random infection. Therefore, the anesthetic concentrations should be determined empirically, and from careful observation of vital parameters and signs of wakefulness, adjusted accordingly during the procedure. Pigs were pre-medicated on medetomidine $(0.4 \mathrm{mg} / \mathrm{kg})$, midazolam $(0.2 \mathrm{mg} / \mathrm{kg})$, and atropine $(0.05 \mathrm{mg} / \mathrm{kg})$ injected intramuscularly. Anesthesia was induced and maintained with propofol/fentanyl. After the day of inoculation, routine urine and blood samples were collected in a deep sedation with a mixture of Zolazepam (10,9 mg/ml), Tiletamin (Zoletil 50 Vet, $50 \mathrm{mg} / \mathrm{ml}$ ), Xylazin (Xyxol Vet, $20 \mathrm{mg} / \mathrm{ml}$ ), Ketamin (Ketaminol $100 \mathrm{mg} / \mathrm{ml}$ ), Butorphanol (Butomidor Vet, $10 \mathrm{mg} / \mathrm{ml}$ ), and Metadon (Comfortan Vet $10 \mathrm{mg} / \mathrm{ml}$ ) as an intramuscular (IM) injection of $1 \mathrm{ml} / 10 \mathrm{~kg}$. The sedation was supplemented after 20-30 min with a dose of $1 \mathrm{ml} / 20 \mathrm{~kg}$, if necessary.

\section{Transurethral Inoculation}

Following anaesthetization, the pigs were placed in a supine position on the operating bed. Both hind legs were raised and moved cranially, and secured with ropes, to expose the urogenital area and making it easier to insert the catheter. After a thorough washing of the urogenital area to remove all visible dirt and debris with warm water and soap, the area was disinfected with two rounds of medical grade iodine solution (7\% iodide) application.

Next, the pigs were catheterized with a sterile Chariérre 10 silicone foley catheter from which urine samples were collected to determine pre-inoculation CFU count. Blood samples were collected in standard EDTA tubes (BD Vacutainer) from the jugular vein.

Following pre-inoculation sampling of urine and blood, the urine content of the bladder was emptied through the catheter. When the flow of the urine ceased, the remaining urine was removed with a large catheter tip syringe, to ensure that any residual urine did not dilute the bacterial suspension to various extend during the subsequent infection procedure. The drained urine volume greatly varied $(50-500 \mathrm{ml})$.

Two sterile $50 \mathrm{ml}$ syringes, with a catheter tip, containing a total of $100 \mathrm{ml}$ inoculum suspension with approximately $10^{8} \mathrm{CFU} / \mathrm{ml}$ were prepared immediately before inoculation (see above). Once the pig was prepared for inoculation, the inoculation suspension was gently infused into the bladder. The process was then repeated with the second syringe. The catheter was then flushed with $10 \mathrm{ml}$ of sterile $0.9 \mathrm{NaCl}$ saline solution, and finally the catheter was clamped using péan forceps (Figure 1A). The inoculum was left in the bladder for $6 \mathrm{~h}$ during which the pig was kept on a restricted IV fluid of $0.5 \mathrm{ml} / \mathrm{min}$ to control urine production. During this period, the vital signs of the pigs were observed carefully. At the 2- and 6-h mark, urine samples from the catheter, and blood samples from the jugular vein, were collected.

\section{Collecting Samples and Monitoring the Infection}

After inoculation, the pigs regularly underwent cystoscopy and biopsies were collected using a Storz C-VIEW video cystoscope (Figure 1B). Urine was collected through a catheter, and blood

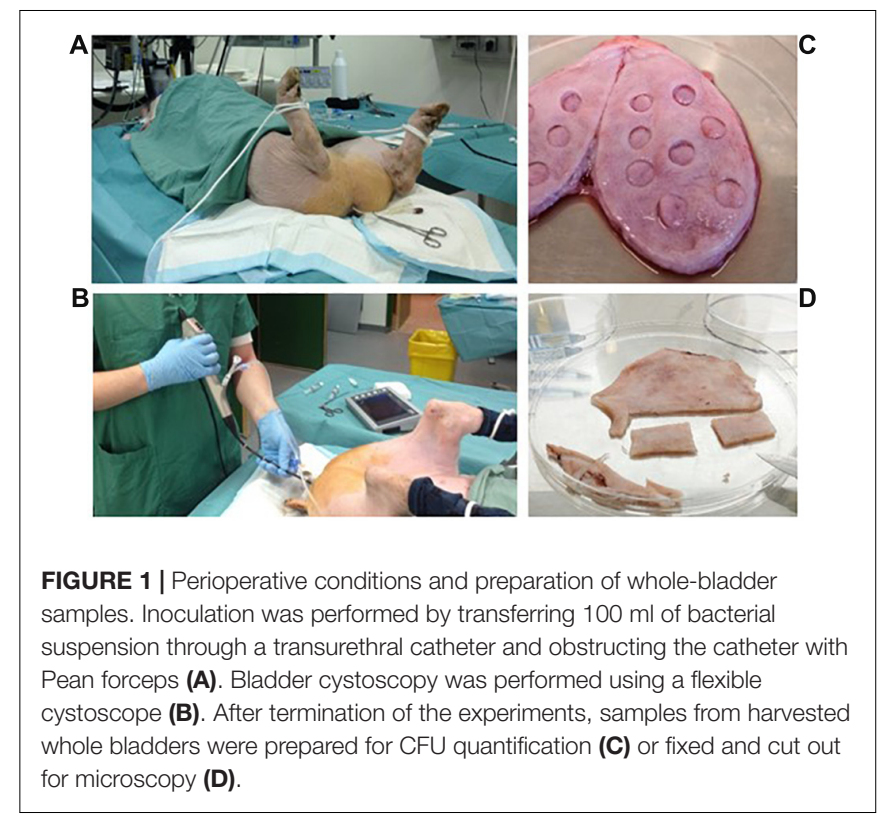

sampled every 2 or 3 days. At the same time points, weight-gain, and rectal temperature were measured.

\section{Sample Analysis}

The level of bacteriuria in urine samples were quantified by serial dilution of urine specimens and plating onto LB agar plates (SSI Diagnostica). Blood samples were analyzed for C-reactive protein (CRP) and for interleukin-6 (IL-6) using Enzyme Linked Immuno-Sorbent Assay (ELISA; Pig CRP ELISA Kit (ab205089) and Pig IL-6 ELISA Kit (ab100755), Abcam). Bladder biopsies obtained during infection were analyzed by Confocal Laser Scanning Microscopy (CLSM, see below) and the number of viable bacteria associated with the tissue estimated by plating of homogenates. The latter was conducted by first dipping the tissue specimens sequentially in three different beakers with sterile saline water to wash off loosely adherent bacterial cells. Then, samples were transferred to plastic tubes containing $5 \mathrm{ml}$ $0.9 \% \mathrm{NaCl}$ saline water, followed by homogenization of the tissue using a rod disperser at $22.000 \mathrm{rpm}$ (IKA Ultra-Turrax T25), serial dilution of the homogenate and plating on LB agar plates (SSI Diagnostica).

\section{Termination}

Pigs were euthanized by sedation with medetomidine $0.04 \mathrm{mg} / \mathrm{kg}$ and midazolam $0.2 \mathrm{mg} / \mathrm{kg}$ IM followed by $5 \mathrm{~mL}$ pentobarbital $(200 \mathrm{mg} / \mathrm{ml}) \mathrm{IV}$. To yield accurate estimates of bacterial numbers in tissue and urine that were not affected by potential postmortem acceleration of bacterial growth, bladder, kidneys, and ureters were surgically removed immediately after termination and transferred under sterile conditions to a laminar air flow cabinet for further processing. Bladders were opened by cutting through one side of the bladder wall in between the ureters by using a scalpel (Figure 1C). Smaller specimens $(\varnothing=1 \mathrm{~cm})$ were punched out of the bladder. Some of these specimens were pinned to a silicone support by using needles and fixed in neutral 


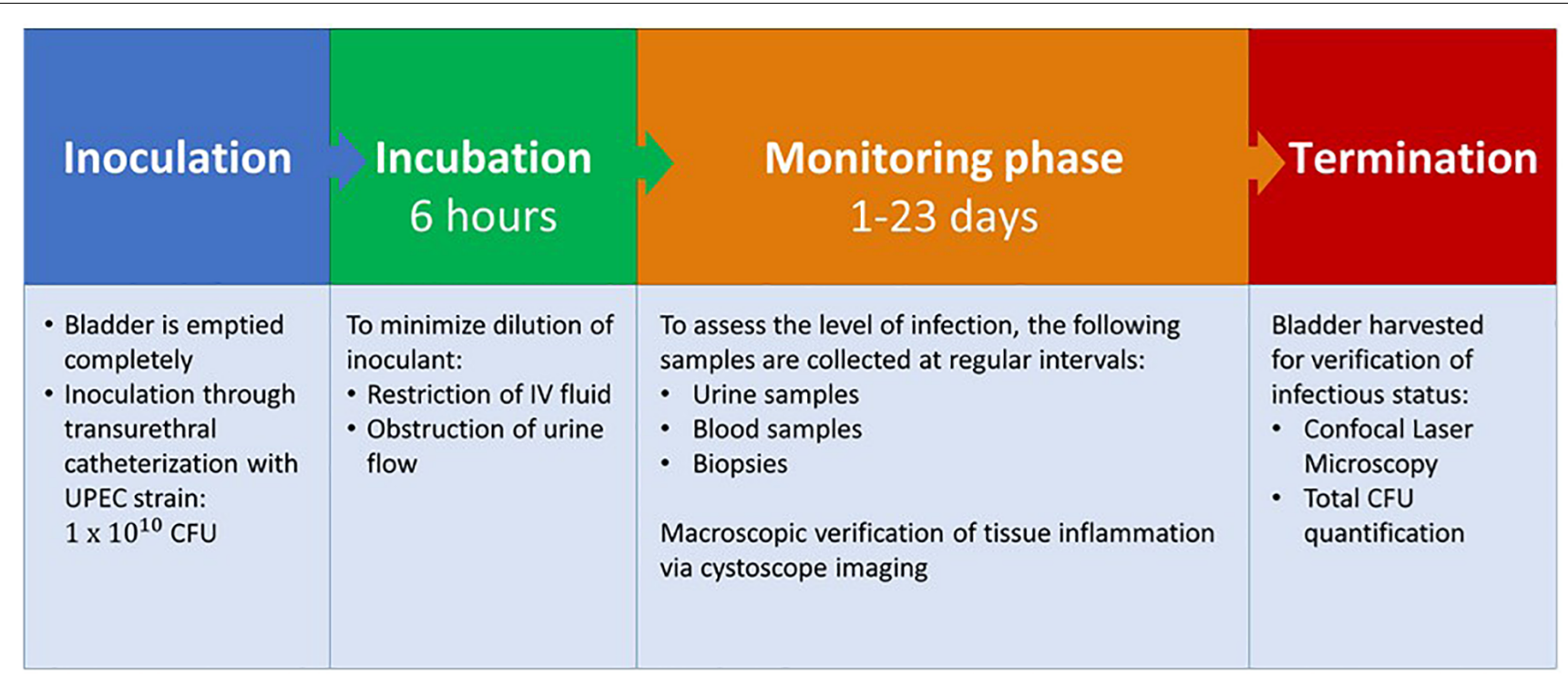

FIGURE 2 | Porcine infection protocol. UPEC, Uropathogenic Escherichia coli; CFU, colony forming units.

buffered formalin solution (Sigma) for later histology (see below). Others were used for CFU quantification as described above for biopsies. For fluorescence microscopy and CLSM, wholebladders were splayed by pinning the bladder in a stretched position onto a silicone support followed by immersion in neutral buffered formalin solution where they were left $\mathrm{ON}$ at $4^{\circ} \mathrm{C}$. Prior to microscopy, $2 \times 4 \mathrm{~cm}$ pieces were cut-out and mounted on microscope slides with the surface facing up (Figure 1D). An overview of the complete porcine infection protocol is summarized in Figure 2.

\section{Confocal Laser Scanning Microscopy}

Fixed and mounted tissue samples were either inspected directly with CLSM for visualization of GFP tagged UTI89, or additionally stained to visualize bladder cell cytoskeleton and cell boundaries. For actin cytoskeleton staining, samples were washed in PBS and bladder cell membranes permeabilized for 5 min with $0.5 \%$ Triton X-100 (Sigma-Aldrich). The tissue specimens were then stained for $30 \mathrm{~min}$ at room temperature with $100 \mathrm{nM}$ Actistain 555 phalloidin (Cytoskeleton, Inc.). CLSM was conducted using an Olympus FV1000MPE CLSM equipped with and an Olympus UPlanSApo $20 \times / 0.85$ objective. Images were captured using the Olympus FV10-ASW software and brightness and contrast were adjusted with the Photoshop Elements software.

\section{Histological Examination}

Formalin fixated tissue samples from the pig bladder wall were cut in $4 \mu \mathrm{m}$ sections using a cryostat and stained with van Gieson-Alcian blue to visualize the urothelium and the associated bacteria. Digital images were obtained using an Olympus BX-60 light microscope fitted with an Evolution MP 5.0 color cooled digital camera (Media Cybernetics). Images were analyzed using Image Pro plus 7.0 software (Media Cybernetics, Silver Spring, MD, United States).

\section{Statistical Analysis}

Statistical analysis was performed with GraphPad Prism 8 software version 8.0. Comparisons were performed with oneway ANOVA followed by Brown-Forsythes test for variance and Tukey's multiple comparisons test. Significance was defined by a $p$-value $<0.05$.

\section{RESULTS}

\section{Significant Bacteriuria Persists in the Porcine Bladder for Prolonged Periods After Inoculation With Uropathogenic Escherichia coli}

An initial question when establishing the model was if the clinical cystitis isolate UTI89 would be able to settle and persist in the porcine bladder after a single inoculation and without any additional intervention. To test this, three groups of two pigs were inoculated with UTI89 and monitored for 12,16 , and 23 days post-inoculation (dpi), respectively. To follow the course of infection, the pigs were sedated biweekly to collect urine and blood samples and to perform cystoscopy and collect biopsies from the bladder. Figure 3 shows that $24 \mathrm{~h}$ post-inoculation (hpi), UTI89 is present in the urine in significant numbers averaging at $10^{7}-10^{8} \mathrm{CFU} / \mathrm{ml}$ (Figure 3C). Analysis of the urine specimens collected at later time points revealed persistent bacteriuria $\left(>10^{5}\right)$ throughout prolonged experiments up to 23 days, indicating establishment of a chronic infection. To visualize macroscopic signs of local inflammation of the bladder, flexible cystoscopy was performed of the pigs revealing an edematous and inflamed bladder mucosa at $24 \mathrm{hpi}$ (Figures 4Aiii,Biii) indicating an ongoing infection in the bladder, analogous to observations in bladders of women with 

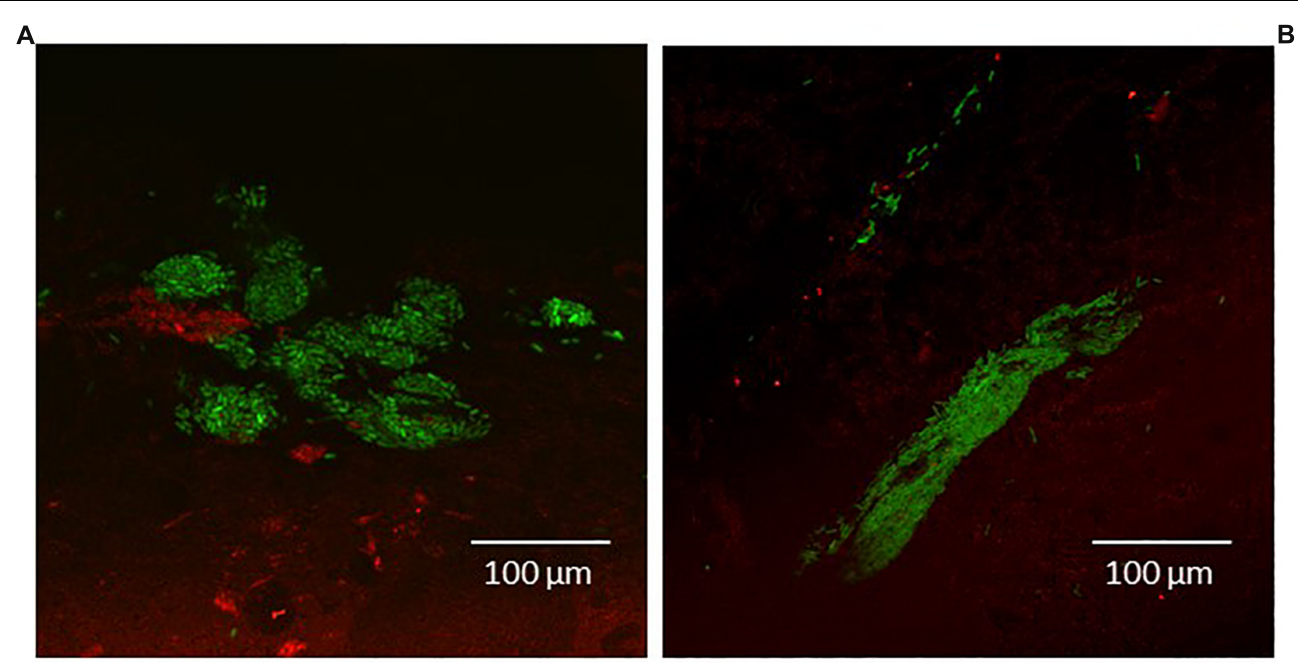

C

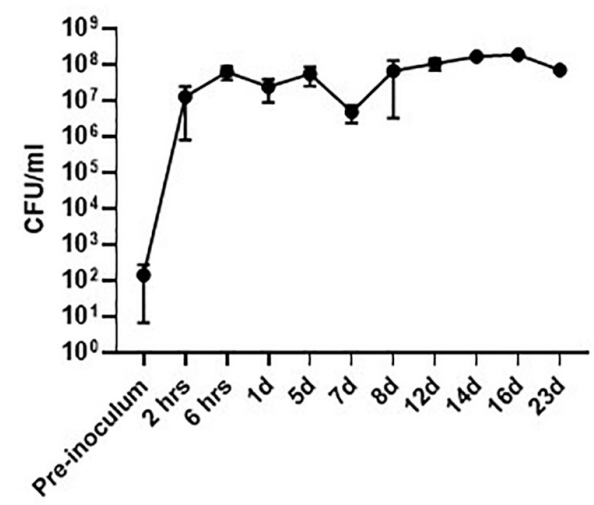

FIGURE 3 | Pigs infected with the green-fluorescent protein-expressing UTI89pMAN01. CLSM of the surface of biopsies performed at $6 \mathrm{~h}$ post-infection (A) and $24 \mathrm{~h}$ post-infection (B) revealed bladder-wall associated bacteria. Urine specimens from infected pigs were analyzed to quantify the level of bacteriuria. $6 \mathrm{~h}$ after inoculation the pigs have developed significant bacteriuria and remains at this level (C). Graph represents data from six pigs \pm standard errors of the mean. Pigs were terminated at 12 days $(n=2), 16$ days $(n=2)$, and 23 days $(n=1)$. The second pig for the 23-day experiment had to be terminated at 5 days due to illness unrelated to the UTI infection. CFU, colony forming units.

recurrent UTI (Santoni et al., 2018). Paradoxical to the persistent bacteriuria observed, the bladder mucosal swelling quickly subsided and appeared habitual at $5 \mathrm{dpi}$ except for pinpoint spots of hemorrhage suggesting that the local inflammation eases off during prolonged infection. CLSM of the bladder wall biopsies showed bacteria localized to discrete patches on the bladder epithelial surface (Figures 3A,B), however, most of biopsy samples revealed little to none bacteria. Due to abundant mucus on the urothelium, suitable biopsies containing epithelium tissue were difficult to obtain, and thus, we refrained from this procedure in the following experiments described.

\section{Inoculation With Uropathogenic Escherichia coli Elicits an Acute and Chronic Inflammatory Response in Infected Pigs}

Significant bacteriuria alone is insufficient to define UTI as bacteriuria may represent an asymptomatic colonization of the bladder (Köves et al., 2017). Although the development of fever and cystoscopy images strongly indicated ongoing infection, we further wanted to correlate this with systemic biomarkers of inflammation. In a separate experiment, four pigs were inoculated with UTI89 and monitored for 12 days with urine and blood sample analysis. The results are presented in Figure 5. Within $24 \mathrm{hpi}$, the pigs developed significant bacteriuria (> $10^{7} \mathrm{CFU} / \mathrm{ml}$ ) (Figure 5A) as the previous experiment. During the initial $24 \mathrm{~h}$, inoculated pigs responded with rapidly elevated granulocytes levels at an average of $14.9 \times 10^{9} / \mathrm{L}$ representing a significant 2 -fold increase from baseline $(p<0.05)$ (Figure 5B), consistent with an infection of bacterial origin. C-reactive protein (CRP), an acute phase reactant associated with infection in humans, behaved correspondingly with more than 7 fold increase from baseline $(p<0.05)$ (Figure 5C; Wagenlehner et al., 2013). At this time point, the pigs also developed hyperthermia with average temperatures of $39,4^{\circ} \mathrm{C}\left( \pm 1,4^{\circ} \mathrm{C}\right)$ compared to the average baseline temperature of $36.4^{\circ} \mathrm{C}$ $\left( \pm 0.8^{\circ} \mathrm{C}\right)$ (Supplementary Figure S1). At $2 \mathrm{dpi}$, granulocytes 


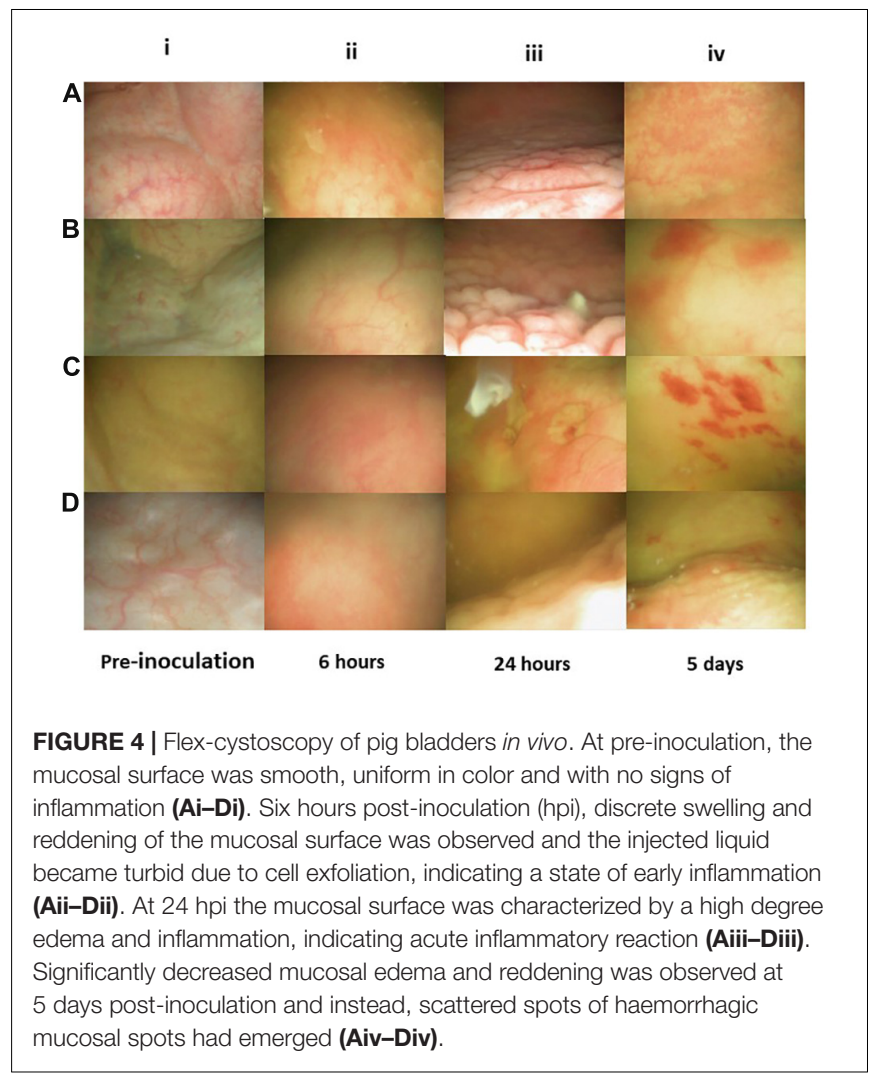

and CRP-levels declined to sub-maximum values (Figures 5B,C). CRP continued to decline reaching baseline levels at $7 \mathrm{dpi}$, whereas granulocytes remained moderately elevated throughout the experiment. Altogether, this suggests a progression from acute inflammation into a chronic infectious stage characterized by an attenuated inflammation. This presumption was further supported by the concordance of significantly decreased bladder mucosal swelling at $5 \mathrm{dpi}$ (Figures 4Civ,Div). The inflammatory biomarker, IL-6 was measured in the blood samples as well, but was found to be unaffected during the course of infection which is, however, in accordance with earlier reports from humans (Benson et al., 1994; Roilides et al., 1999).

\section{UPEC Permanently Colonize the Bladder Epithelial Mucosa}

We sought to investigate the magnitude and morphology of epithelial colonization during infection in our porcine model. Thus, we performed a separate experiment in two pigs that were infected with the green fluorescent protein-expressing UTI89pMAN01 and terminated after $12 \mathrm{~h}$ to allow detailed analysis of the progression of colonization at the early state of the infection. Harvested whole bladders from terminated pigs were analyzed for localization and morphology of bacteria using CLSM and histological examination. CLSM showed that large amounts of bacteria were associated with the bladder epithelial mucosa. The bacteria were mainly localized in discrete areas on the bladder luminal surface, rather than distributed randomly on the bladder epithelium (Figure 6A). In between these areas were large areas without any detectable bacteria. Areas with surface-associated bacteria appeared inflamed and were characterized by swollen epithelium with deep pockets that harbored the majority of bacteria in these regions (Figure 6B). At several sites, uroepithelial cells were covered extensively by bacteria that appeared embedded in biofilm (Figure 6C). Histological examination further supported the presence of a surface-associated UPEC (Figure 6D). To quantify epithelial colonization, harvested whole bladder cut-outs from infected pigs were washed in PBS, homogenized, and plated on agar to quantify the number of bacteria associated to the bladder mucosal surface. The results are displayed in Figure $6 \mathrm{E}$ showing significant bacterial loads $\left(10^{4}-10^{7} \mathrm{CFU} / \mathrm{g}\right)$ at every time-point suggesting persistent epithelial colonization. Homogenates of kidney tissue-sections were sterile (data not shown), confirming that the infection was confined to the bladder without ascending from the urinary tract. Homogenization of ureteral tissue proved infeasible, and thus, no CFU data is available from these samples.

\section{DISCUSSION}

Given that UTI remains a considerable challenge at hospitals and in the community, it is critical that the considerable fundamental biological investigation conducted over the past decades is translated into actual novel treatment regimens. A key step to achieve this is the verification that previous results obtained from in vitro and small-animal models also reflect the infection pathogenesis in humans.

Although murine studies have elevated greatly our understanding of general UPEC UTI infection pathogenesis, the general translatability of in vitro and murine infection research to human infection is still being questioned in the infection research society. In addition to the major differences in urogenital size and anatomy of mice and humans, and general immune response (Dawson et al., 2017), the highly concentrated nature of mouse urine, compared to the urine of larger mammals such as humans and pigs, is another concern to their frame of reference, since urine concentration is shown to directly influence both UPEC biofilm capacity and UPEC cell morphology, both of which has been linked to virulence in the urinary tract (Parfentjev and Perlzweig, 1932; Justice et al., 2006; Andersen et al., 2012; Klein et al., 2015; Subashchandrabose and Mobley, 2015). In drug development, concerns are also being raised about the use of murine models, as highlighted in a recent report stating that $>80 \%$ of therapeutic treatments that were safe and effective in animal models, failed to succeed in human trials (Perrin, 2014). These challenges with animal models for understanding pathogenesis and predicting therapeutic outcome in the human host, emphasize the need to improve pre-clinical models to better reproduce the various aspects of disease in humans.

To accommodate this need, we here present a large animal model of cystitis, one of the most frequent infectious diseases in humans. We chose to use pigs as model animal, since they are more similar to humans with respect to urogenital anatomy, urine density, immune system physiology, and furthermore is a natural host of UPEC that recapitulates important aspects of 


\section{A}

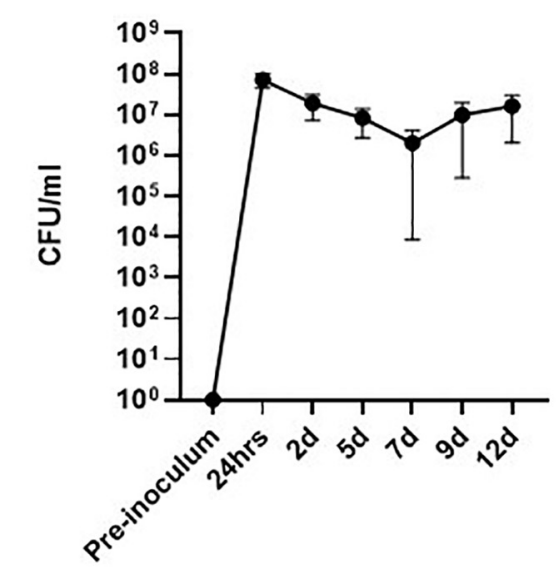

C

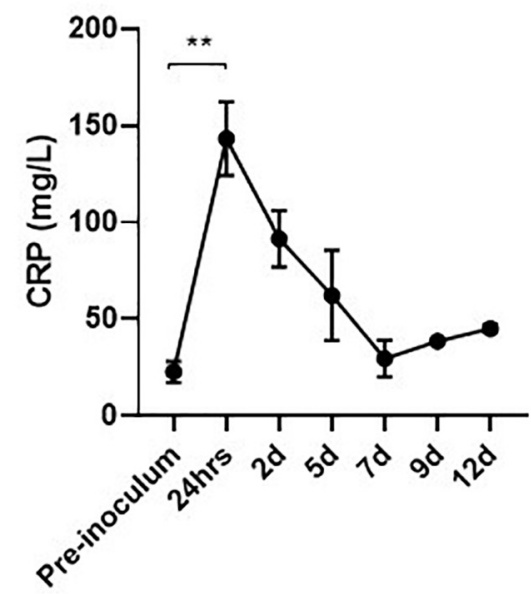

B

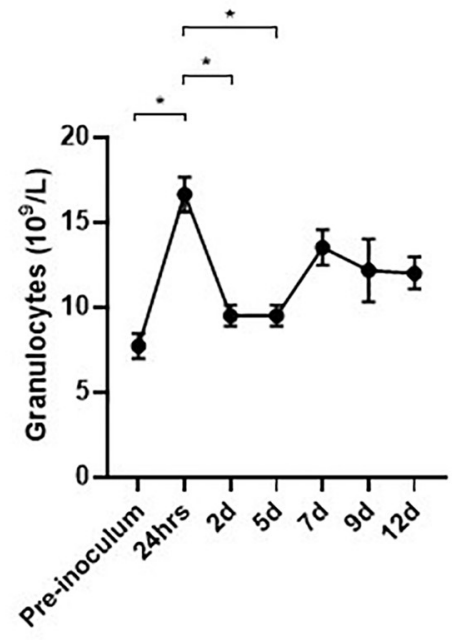

D

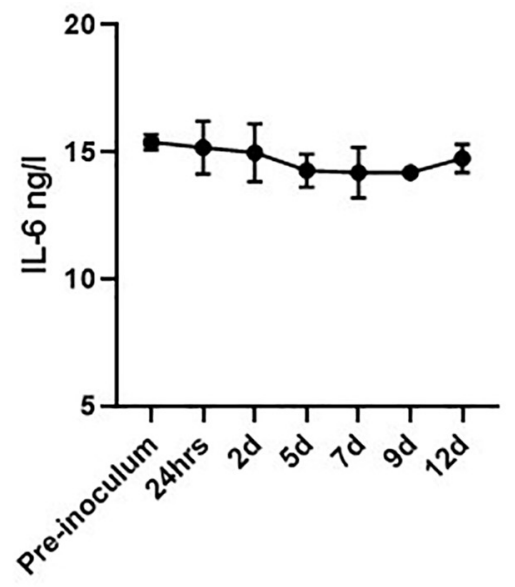

FIGURE 5 | Level of bacteriuria and inflammatory biomarkers during infection of pigs $(n=4)$. The infected pig bladders remain significantly colonized throughout the experiment (A). Within the first $24 \mathrm{~h}$ of infection, granulocytes are rapidly rising reaching maximum concentrations of $14.9 \times 10^{9} / \mathrm{ml}$. Shortly after, a small drop in granulocyte level is observed though remaining elevated until termination of the experiment (B). CRP increases $>7$-fold at 24 hpi (peak-value) and decrease linearly hereafter, reaching baseline values at $7 \mathrm{dpi}$ (C). No observable change in levels of IL-6 was seen during infection (D). Graphs represent means \pm standard error of the mean. ${ }^{*} P<0.05,{ }^{*} P<0.001$, one-way ANOVA with Tukey's multiple comparisons test. Data from 7 days onward are based on two pigs only. CFU, colony forming units; IL-6, interleukin-6.

human UTI (Meurens et al., 2012; Barber, 2016). To date, UTI in pigs is limited to a few studies investigating pathology and renal scarring in relation to surgically induced acute pyelonephritis, and to our knowledge, no porcine model exists for establishing a persistent non-ascending UTI (Coulthard et al., 2002; Farhat et al., 2002; Isling et al., 2011; Barber, 2016). Although a non-human primate model of lower UTI with vervet monkeys has been described, a pig model is a feasible alternative due to global availability of animals and lighter ethical concerns (Hyre et al., 2017).

In the porcine model presented here, a single transurethral inoculation with UPEC is sufficient to induce a bladder infection. The pressure generated by transurethral inoculation of $100 \mathrm{ml}$, could raise concerns about vesicourethral reflux resulting in bacteria reaching the ureters and kidneys, as this has initially proven challenging to avoid in murine models (Schaeffer et al.,
1987; Hopkins et al., 1995; Hung et al., 2009). In the porcine model, however, sterile homogenates of kidney tissue samples from infected pigs, indicated that no such bacterial ascension has occurred during inoculation of the pigs. The UPEC strain UTI89 used as infectious agent was originally isolated from a nonascending cystitis in a human and our results indicate a similar infection symptomatology in the pig (Mulvey et al., 2001). Since the porcine kidney specimens were extracted at the termination of the experiment ( $>5$ days), we can, however, not rule out the possibility that bacteria may have reached the upper urinary tract immediately after inoculation, although insufficient to colonize these sites persistently.

In response to bacterial inoculation, the pigs mustered an inflammatory response of fever, bladder mucosal edema, elevated granulocytes and CRP, all characteristics of UTI in the human host (Wagenlehner et al., 2013; Gupta et al., 2017; Santoni et al., 


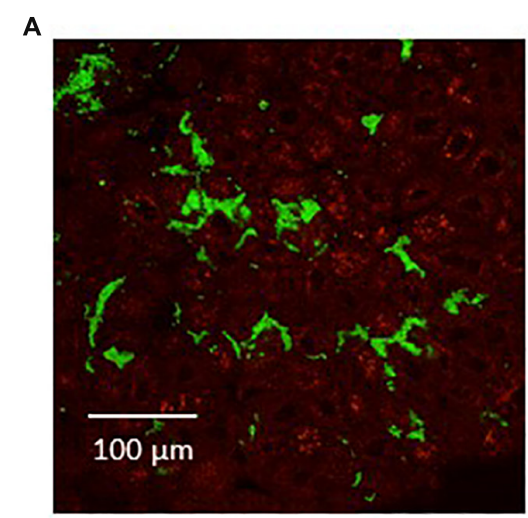

B

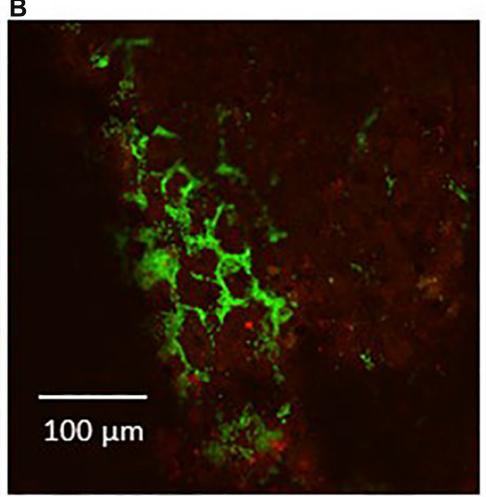

D
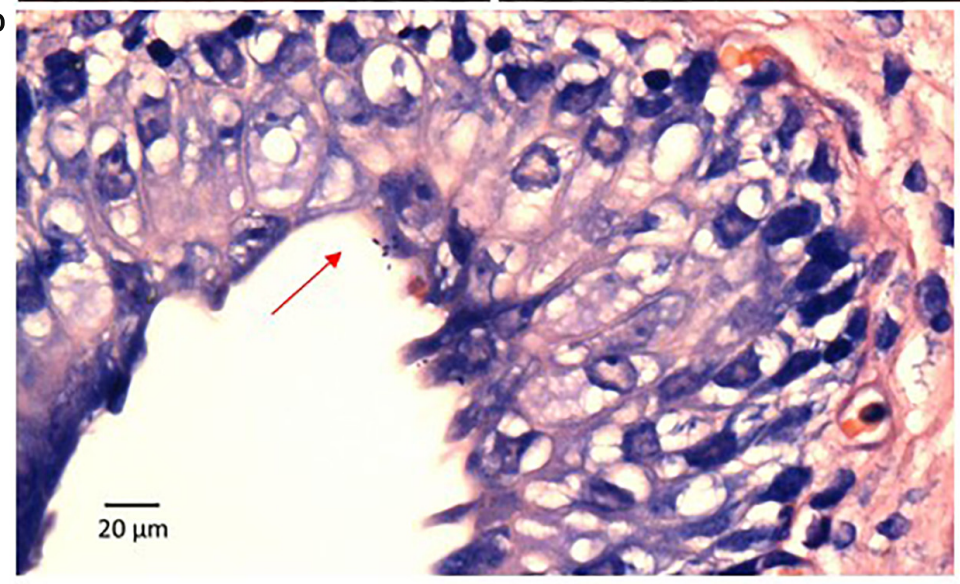

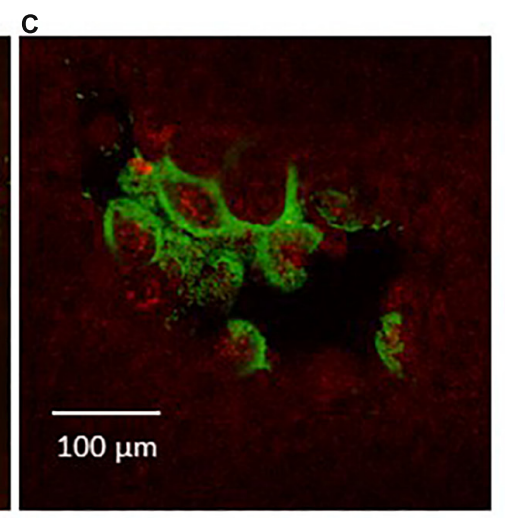

E

Days post infection

FIGURE 6 | Confocal laser scanning microscopy of the surface of whole bladder sections after $12 \mathrm{~h}$ infection with the green fluorescent protein-expressing UTI89pMAN01. Large amounts of bacteria (green) were associated with the bladder tissue (red) although they were found in specific areas of swollen bladder epithelium (A). Large areas in between these infected areas were almost completely devoid of bacteria. The bacteria in infected areas were primarily located in deep pockets of the uroepithelium and inspection at higher magnification revealed that bacteria in general appeared localized to the boarders between the uroepithelial cells (B). At several sites, uroepithelial cells were covered in bacteria that were growing in biofilm-like structures (C). Histological examination revealed surface associated bacteria as well (D), although the above described distribution of bacteria made it difficult with this method to localize colonized sites (D). Whole bladder sections that were washed in PBS, homogenized and plated, showed significant quantities of bacteria associated with the bladder mucosa that persisted for prolonged infection experiments $\mathbf{( E )}$. Bars represents means \pm standard errors of the mean. ${ }^{*} P<0.01$, ${ }^{* *} P<0.0001$, one-way ANOVA with Tukey's multiple comparisons test. CFU, colony forming units.

2018). The onset of ongoing bacteriuria, increased CRP and granulocytes levels also reflect observations from a UTI model with non-human primates (Hyre et al., 2017). After 24 h postinoculation, the acute infection progressed into a chronic stage characterized by reduced swelling of the bladder mucosa and submaximum values of infectious biomarkers. This inflammatory response confirms that the bacteriuria observed is indeed an infection, and not a result of asymptomatic bacteriuria, as is otherwise commonly seen in human patients (Köves et al., 2017). In recent years, the inflammatory cytokine, IL-6, has been investigated as a potential urine biomarker for discriminating between asymptomatic bacteriuria and UTI and has recently been associated with the severity of symptoms in non-febrile lower UTI (Sundén et al., 2017). Increased serum IL-6 levels, on the other hand, has mainly been associated to febrile patients with pyelonephritis (Otto et al., 1999; Gürgöze et al., 2005; Sheu et al., 2006). Also, in studies of neonates and children, urine IL-6 was significantly higher among UTI patients compared to patients without bacteriuria, whereas serum IL-6 did not differ (Benson et al., 1994; Roilides et al., 1999). In the porcine model, no significant change in serum IL-6 was detected during the course of infection, further suggesting that serum IL-6 may be a less promising biomarker compared to urine IL-6 for diagnosing lower UTI. In this study, urine IL-6 was not assessed due to unavailable ELISA reagents. Compared to rodent models, the restricted selection of commercially available porcine reagents is a general limitation of the model to study hostpathogen interactions.

Within the first $24 \mathrm{~h}$ post-inoculation, and in agreement with standard diagnostic criteria in humans, the pigs develop significant bacteriuria (> $10^{5} \mathrm{CFU} / \mathrm{ml}$ ) (Scott et al., 2015). Also, the significant level of bacteriuria persists for prolonged experiments up to 23 days. To survive in the bladder for such a prolonged time, UPEC must be able to successfully circumvent inflammatory host-responses and hydrodynamic flushing by the flow of urine. The latter is likely attributable to a strong 
epithelial attachment facilitated by various adhesive fimbriae (Connell et al., 1996; Thomas et al., 2004; Jorgensen and Seed, 2012). Here, we show the existence of a significant epithelial colonization in vivo with bacterial titers of $10^{4}-10^{7} \mathrm{CFU} / \mathrm{g}$. This in agreement with a previous study, in which we showed that during infection in vitro, UPEC establishes as a sessilepopulation on the surface of human urothelial cell layers exposed to a urine hydrodynamic environment (Andersen et al., 2012). Although prone to elimination by urine flushing, bacterial cells released from these sessile populations in vivo, may disseminate the infection to uninfected areas of the bladder mucosa as well as other sites of the urinary tract, and this changeable balance between sessile and planktonic growth forms is likely to ensure persistent survival of UPEC in the bladder. Indeed, significant loads of surface-associated bacteria were present in the porcine bladders for prolonged infection for up to 23 days suggesting that the bladder surface is a main habitat for establishing a persistent population in the bladder. Rather than random distribution of individually attached bacteria, CLSM and histological examination revealed that sessile UPEC establish on the bladder luminal surface as small colonies or in biofilmlike aggregates. Prior to this study, the sessile presence and distribution in the urinary bladder has to our knowledge never been assessed in vivo and we were surprised to find, that attached bacteria were absent on large areas of the bladder surface. The relatively sparse areas of sessile bacterial presence is a plausible explanation to the lacking bacteria in most biopsied tissue-samples. In future studies, the value of biopsies should be carefully considered, in light of the practical challenges and animal stress involved with the procedure.

In comparison to humans and pigs, mice are extremely resistant to endotoxin shock and responds to infection with hypothermia rather than hyperthermia (Meurens et al., 2012). This is limiting for using mice as models for studying moderate to severe infections associated with hyperthermia. Indeed, a significant characteristic of the porcine-model presented here is the development of moderate fever $\left(39-40^{\circ} \mathrm{C}\right)$ during the acute infectious phase. Although fever is not always present in cases of uncomplicated UTI in humans, the absence of bacteria in the kidney tissue homogenates shows that the cystitis isolate UTI89 used here only induces a local infection confined to the porcine bladder, and thus, the fever observed was not due to bacterial ascension in the urinary tract and translocating of bacteria to the blood. This was further supported by the weight gains of the pigs, which were as expected of their age and indicating that they thrived and were most likely not subject to systemic infection and sepsis. It is more likely that the hyperthermia observed was a sign of a massive acute inflammatory process in response to the relatively large bacterial inoculum concentration of $10^{8} \mathrm{CFU} / \mathrm{ml}$. This inoculum concentration was chosen based on what is traditionally used in murine UTI models, where it is required for successful infection, and hence would allow for direct comparison between these two animals (Hung et al., 2009). However, in contrast to mice, pigs and humans are natural hosts of UPEC and naturally susceptible to UTI and therefore this inoculum is presumably larger than necessary for establishing a UTI in these species. Ongoing studies indicate that significantly lower inoculum concentrations are adequate to cause UTI in the pig (data not shown) and hence, could be used in case no direct comparison are made to the mouse. Further studies are needed to uncover the minimal bacterial load necessary for colonizing the porcine urinary tract for optimal comparison to the course of infection in humans, and due to the above reasons, such studies cannot be conducted in rodent models and require large animal models.

Despite the localized infection presented here, the ability of inducing hyperthermia in the porcine model is a quality that grants the potential of studying disseminated infections and urosepsis, a life-threatening complication to infections originating from the urinary tract and one of the most common cause of septicemia (Wagenlehner et al., 2008; Bonkat et al., 2018). To our knowledge, only one study to date, has documented urosepsis in pigs using porcine-isolated pyelonephritis strains, however, in light of the high prevalence of urosepsis in human patients, studies using human pathogenic strains are warranted (Isling et al., 2011).

An extensively studied pathogenic component in UPEC UTI is the type-1 fimbriae (Hagan et al., 2010; Jorgensen and Seed, 2012; Stærk et al., 2016). This adherence fimbrium is critical for murine UTI (Connell et al., 1996; Martinez et al., 2000) and thus believed to play a role in human UTI as well (Connell et al., 1996). Yet, solid evidence is lacking that demonstrates a major role of this fimbria in the pathogenesis of human cystitis (Johnson, 1991; Bower et al., 2005; Bergsten et al., 2007; Jorgensen and Seed, 2012). The down-regulation of type- 1 fimbriae in the urine of UTI patients raises questions regarding its significance for human UTI (Hagan et al., 2010; Subashchandrabose et al., 2014) although in vitro modeling of infection indicates that this apparent paradox might be explained by expression of the fim genes only in surface-associated populations (Stærk et al., 2016).

The inoculation suspension of UTI89 used here, was pregrown under circumstances that increases type- 1 fimbriae expression which is standard protocol used in the murine UTI model to facilitate successful infection (Hung et al., 2009). To which extend this artificial induction of type-1 fimbriae affects the course of infection in the pigs is, however, unknown, but a subject for future research in our group. Previous experimental studies of acute pyelonephritis in pigs showed an increased severity of histological lesions in kidneys after infection with strains expressing both type-1 fimbriae and P-fimbriae suggesting that these fimbriae contribute to UPEC virulence during ascending UTI in pigs (Isling et al., 2011). However, the importance of type-1 fimbriae for non-ascending UTI in pigs remains unknown. Further large-animal studies with type-1 pili attenuated strains are needed to elucidate the significance of this virulent factor during UTI.

In conclusion, a method is presented here that induces persistent cystitis in a large animal model of cystitis thus permitting the study of UPEC pathogenesis in a urinary tract with a physiology comparable to humans. The model development aimed to provide an experimental platform that can help translate decades of extensive UTI research from in vitro and murine experiments into clinical therapeutic strategies. The model described, facilitates the study of both acute and chronic 
UPEC pathogenic mechanisms and host immune effectors relevant to UTI and offers the stepping stone for studying ascending and invasive infections as well. Additional studies in this setup will furthermore enable investigation of current UTI pathogenesis paradigms derived from the murine model, such as intracellular colonization and phenotypic plasticity as possible major determinants of UPEC virulence and persistence (Mulvey et al., 2001; Justice et al., 2008).

\section{DATA AVAILABILITY STATEMENT}

The raw data supporting the conclusions of this manuscript will be made available by the authors, without undue reservation, to any qualified researcher.

\section{ETHICS STATEMENT}

This study was carried out in accordance with the recommendations of the Danish Animal Experiment Inspectorate. The protocol was approved by the Danish Animal Experiment Inspectorate, license number: 2017-15-0201-01271.

\section{AUTHOR CONTRIBUTIONS}

TN, NP, RG, TA, and LL conceived the study idea and design. TN, NP, YP, RG, TA, LN, and LL performed the experimental

\section{REFERENCES}

Andersen, T. E., Khandige, S., Madelung, M., Brewer, J., Kolmos, H. J., and MøllerJensen, J. (2012). Escherichia coli uropathogenisis in vitro: invasion, cellular escape, and secondary infection analyzed in a human bladder cell infection model. Infect Immun. 80, 1858-1867. doi: 10.1128/IAI.06075-11

Barber, A. E. (2016). Strenght and limitations of model systems for the study of Urinary tract infections and realated pathologies. Microbiol. Mol. Biol. Rev. 80, 351-367. doi: 10.1128/MMBR.00067-15

Benson, M., Jodal, U., Andreasson, A., Karlsson, A., Rydberg, J., and Svanborg, C. (1994). Interleukin 6 response to urinary tract infection in childhood. Pediatr Infect Dis J. 13, 612-616. doi: 10.1097/00006454-19940700000006

Bergsten, G., Wullt, B., Schembri, M. A., Leijonhufvud, I., and Svanborg, C. (2007). Do type 1 fimbriae promote inflammation in the urinary tract? Cell Microbiol. 9, 1766-1781. doi: 10.1111/j.1462-5822.2007. 00912.x

Bielecki, P., Muthukumarasamy, U., Eckweiler, D., Bielecka, A., Pohl, S., Aschanz, A., et al. (2014). In vivo mRNA profiling of uropathogenic Escherichia coli from diverse phylogroups reveals common and groupspecific gene expression profiles. Mbio 5:e1075-14. doi: 10.1128/mBio. 01075-14

Bjerklund Johansen TE, Cek, M., Naber, K., Stratchounski, L., Svendsen, M. V., Tenke, P., et al. (2007). Prevalence of hospital-acquired urinary tract infections in urology departments. Eur. Urol. Apr. 51, 1100-1111.

Bonkat, G., Cai, T., Veeratterapillay, R., Bruyére, F., Bartoletti, R., Pilatz, A., et al. (2018). Management of urosepsis in 2018. Eur. Urol. Focus. 5, 5-9.

Bower, J. M., Eto, D. S., and Mulvey, M. A. (2005). Covert operations of uropathogenic Escherichia coli within the urinary tract. Traffic 6, 18-31. doi: 10.1111/j.1600-0854.2004.00251.x work. KS completed the first draft of the manuscript. TN and TA contributed to sections of the manuscript. All authors were involved in the discussion and interpretation of the results, contributed to manuscript revision, and has read and approved the submitted version.

\section{FUNDING}

This study was supported by the Region of Southern Denmark, project grant number 17/33837, Beckett Fonden, and Coloplast A/S.

\section{ACKNOWLEDGMENTS}

The authors would like to thank the personnel of the Central Animal Facility, the Biomedical Laboratory of Odense University Hospital for their technical support. A special thanks goes to Lise Christensen from Coloplast A/S for assisting with histological examination.

\section{SUPPLEMENTARY MATERIAL}

The Supplementary Material for this article can be found online at: https://www.frontiersin.org/articles/10.3389/fmicb. 2019.02564/full\#supplementary-material

Cai, T., Verze, P., Brugnolli, A., Tiscione, D., Luciani, L. G., Eccher, C., et al. (2016). Adherence to European association of urology guidelines on prophylactic antibiotics: an important step in antimicrobial stewardship. Eur .Urol. 69, 276-283. doi: 10.1016/j.eururo.2015. 05.010

Carey, A. J., Tan, C. K., Ipe, D. S., Sullivan, M. J., Cripps, A. W., Schembri, M. A., et al. (2016). Urinary tract infection of mice to model human disease: practicalities, implications and limitations. Crit. Rev. Microbiol. 42, 780-799. doi: 10.3109/1040841X.2015.1028885

Connell, H., Agace, W., Klemm, P., Schembri, M., Mårild, S., and Svanborg, C. (1996). Type 1 fimbrial expression enhances Escherichia coli virulence for the urinary tract. Proc. Natl. Acad. Sci. U.S.A. 93, 9827-9832.

Coulthard, M. G., Flecknell, P., Orr, H., Manas, D., and O’Donnell, M. (2002). Renal Scarring caused by vesicoureteric reflux and urinary infection: a study in pigs. Pediatric Nephrol. 17, 481-484. doi: 10.1007/s00467-0020878-2

Dawson, H. D., Smith, A. D., Chen, C., and Urban, J. F. Jr. (2017). An in-depth comparison of the porcine, murine and human inflammasomes; lessons from the porcine genome and transcriptome. Vet. Microbiol. 202, 2-15. doi: 10.1016/ j.vetmic.2016.05.013

European Center for Disease Control, and Prevention [ECDC], (2013). Point Prevalence Survey of Healthcare Associated Infections and Antimicrobial Use in European Acute Care Hospitals, 2011-2012. Stockholm: European Center for Disease Control and Prevention.

Farhat, W., Traubici, J., Sherman, C., Williams, T., Babyn, P., and McLorie, G. (2002). Reliability of contrast enhanced sonography with harmonic imaging for detecting early renal scarring in experimental pyelonephritis in a porcine model: preliminary results. J. Urol. 168, 1114-1117. doi: 10.1097/00005392-20020900000066 
Foxman, B. (2014). Urinary tract infection syndromes: occurrence, recurrence, bacteriology, risk factors, and disease burden. Infect Dis. Clin. North Am. 28, 1-13. doi: 10.1016/j.idc.2013.09.003

Foxmann, B. (2010). The epidemiology of urinary tract infection. Nat. Rev. Urol. 7, 653-660. doi: 10.1038/nrurol.2010.190

Guglietta, A. (2017). Recurrent urinary tract infections in women: risk factors, etiology, pathogenesis and prophylaxis. Future Microbiol. 12, 239-246. doi: 10.2217/fmb-2016-0145

Gupta, K., Grigoryan, L., and Trautner, B. (2017). Urinary tract infection. Ann. Intern. Med. 167, ITC49-ITC64. doi: 10.7326/AITC2017 10030

Gürgöze, M. K., Akarsu, S., Yilmaz, E., Gödekmerdan, A., Akca, Z., Ciftci, I., et al. (2005). Proinflammatory cytokines and procalcitonin in children with acute pyelonephritis. Pediatr Nephrol. 20, 1445-1448. doi: 10.1007/s00467-0051941-6

Hagan, E. C., Lloyd, A. L., Rasko, D. A., Faeber, G. J., and Mobley, H. L. (2010). Escherichia coli global gene expression in urine from women with urinary tract infection. PLoS Pathog 6:e1001187. doi: 10.1371/journal.ppat.100 1187

Hopkins, W. J., Hall, J. A., Conway, B. P., and Uehling, D. T. (1995). Induction of urinary tract infection by intraurethral inoculation with Escherichia coli: refining the murine model. J. Infect Dis. 171, 462-465. doi: 10.1093/infdis/171. 2.462

Hung, C. S., Dodson, K. W., and Hultgren, S. J. (2009). A murine model of urinary tract infection. Nat. Protoc. 4, 1230-1243. doi: 10.1038/nprot. 2009.116

Hyre, A. N., Kavanagh, K., Kock, N. D., Donati, G. L., and Subashchandrabose, S. (2017). Copper is a host effector mobilized to urine during urinary tract infection to impair bacterial colonization. Infect Immun. 85:e1041-16. doi: 10. 1128/IAI.01041-16

Isling, L. K., Aalbæk, B., Birck, M. M., Heegaard, P. M., and Leifsson, P. S. (2011). Host response to porcine strains of Escherichia coli in a novel Pyelonephritis Model. J. Comp. Pathol. 144, 257-268. doi: 10.1016/j.jcpa.2010. 10.002

Johnson, J. R. (1991). Virulence factors in Escherichia coli urinary tract infection. Clin. Microbiol. Rev. 4, 80-128. doi: 10.1128/cmr.4.1.80

Jorgensen, I., and Seed, P. C. (2012). How to make it in the urinary tract: a tutorial by Escherichia coli. PLoS Pathog 8:e1002907. doi: 10.1371/journal.ppat.100 2907

Justice, S. S., Hunstad, D. A., Cegelski, L., and Hultgren, S. J. (2008). Morphological plasticity as a bacterial survival strategy. Nat. Rev. Microbiol. 6, 162-168. doi: $10.1038 /$ nrmicrol 1820

Justice, S. S., Lauer, S. R., Hultgren, S. J., and Hunstad, D. A. (2006). Maturation of intracellular Escherichia coli communities requires SurA. Infect Immun. 74, 4793-4800. doi: 10.1128/iai.00355-06

Khandige, S., Antoinette Asferg, C., Rasmussen, K. J., Larsen, M. J., Overgaard, M., Andersen, T. E., et al. (2016). DamX controls reversible cell morphology switching in uropathogenic Escherichia coli. mBio 7:e00642-16. doi: 10.1128/ mBio.00642-16

Klein, K., Palarasah, Y., Kolmos, H. J., Møller-Jensen, J., and Andersen, T. E. (2015). Quantification of filamentation by uropathogenic Escherichia coli during experimental bladder cell infection by using semi-automated image analysis. J. Microbiol. Methods 109, 110-116. doi: 10.1016/j.mimet.2014. 12.017

Köves, B., Cai, T., Veeratterapillay, R., Pckard, R., Seisen, T., Lam, T. B., et al. (2017). Benefits and harms of treatment of asymptomatic bacteriruria: a sytematic revew and metaanalysis by the Europea Association of Urology Urological infection guidelines panel. Eur. Urol. 72, 865-868. doi: 10.1016/j. eururo.2017.07.014

Magill, S. S., Edwards, J. R., Bamberg, W., Beldavs, Z. G., Dumyati, G., Kainer, M. A., et al. (2014). Multistate point-prevalence survey of health careassociated infections. N. Engl. J. Med. 370, 1198-1208. doi: 10.1056/NEJMoa130 6801

Martinez, J. J., Mulvey, M. A., Schilling, J. D., Pinkner, J. S., and Hultgren, S. J. (2000). Type 1 pilus-mediated bacterial invasion of bladder epithelial cells. EMBO J. 19, 2803-2812. doi: 10.1093/emboj/19.12. 2803
Meurens, F., Summerfield, A., Nauwynck, H., Saif, L., and Gerdts, V. (2012). The Pig: a model human infectious disease. Trends Microbiol. 20, 50-57. doi: 10.1016/j.tim.2011.11.002

Mulvey, M. A., Schilling, J. D., and Hultgren, S. J. (2001). Establishment of a persistent Escherichia coli reservoir during the acute phase of a bladder infection. Infect Immun. 69, 4572-4579. doi: 10.1128/iai.69.7.4572-4579. 2001

Otto, G., Braconier, J., Andreasson, A., and Svanborg, C. (1999). Interleukin6 and desease severity in patients with bacteremia and nonbacteremic febrile urinary tract infection. J. Infect Dis. 179, 172-179. doi: 10.1086/ 314534

Parfentjev, I., and Perlzweig, W. (1932). The composition of the urine of white mice. J. Biol. Chem. 100, 551-555.

Pedersen, R. M., Grønnemose, R. B., Stærk, K., Asferg, C. A., Andersen, T. B., Kolmos, H. J., et al. (2018). A method for quantification of epithelium colonization capacity by pathogenic bacteria. Front. Cell. Infect. Microbiol. 8:16. doi: $10.3389 /$ fcimb.2018.0016

Perrin, S. (2014). Preclinical research: make mouse studies work. Nature 507, 423-425. doi: 10.1038/507423a

Roilides, E., Papachristou, F., Gioulekas, E., Tsaparidou, S., Karatzas, N., Sotiriou, J., et al. (1999). Increased urine interleukin 6 concentrations correlate with pyelonephritic changes on $99 \mathrm{mTc}$-dimercaptosuccinic acid scans in neonates with urinary tract infections. J. Infect Dis. 180, 904-907. doi: 10.1086/ 314960

Santoni, N., Ng, A., Skews, R., and Aoumarzouk, O. M. (2018). Recurrent urinary tract infections in women: what is evidence for investigating with flexible cystoscopy. Imaging and Urodynamics? Urol. Int. 101, 373-381. doi: 10.1159/ 000490918

Schaeffer, A. J., Schwan, W. R., Hultgren, S. J., and Duncan, J. L. (1987). Relationship of type 1 pilus expression in Escherichia coli to ascending urinary tract infection in genetically distinct mouse strains. Infect Immun. 55, 373-380.

Scott, V. S. C., Haake, D. A., Churchill, B. M., Justice, S. S., and Kim, J. (2015). Intracellular bacterial communities: a potential etiology for chronic lower urinary tract symptoms. Urology 86, 425-431. doi: 10.1016/j.urology.2015.04. 002

Seok, J., Warren, H. S., Cuenca, A. G., Mindrinos, M. N., Baker, H. V., Xu, W., et al. (2013). Genomic responses in mouse models poorly mimic human inflammatory diseases. Proc. Natl. Acad. Sci. U.S.A. 110, 3507-3512. doi: 10. 1073/pnas.1222878110

Sheu, J. N., Chen, M. C., Lue, K. H., Cheng, S. L., Lee, I. C., Chen, S. M., et al. (2006). Serum and urine levels of interleukin-6 and interleukin-8 in children with acute pyelonephritis. Cytokine 36, 276-282. doi: 10.1016/j.cyto.2007. 02.006

Soto, S. M., Smithson, A., Horcajada, J. P., Martinez, J. A., Mensa, J. P., and Vila, J. (2006). Implication of biofilm formation in the persistence of urinary tract infection caused by uropathogenic Escherichia coli. Clin. Microbiol. Infect. 12, 1034-1036. doi: 10.1111/j.1469-0691.2006. 01543.x

Stærk, K., Khandige, S., Kolmos, H. J., Møller-Jensen, J., and Andersen, T. E. (2016). Uropathogenic Escherichia coli express type 1 fimbriae only in surface adherent populations under physiological growth conditions. J. Infect Dis. 213, 386-394. doi: 10.1093/infdis/jiv422.v

Subashchandrabose, S., Hazen, T. H., Brumbaugh, A. R., Himpsl, S. D., Smith, S. N., Ernst, R. D., et al. (2014). Host-specific induction of Escherichia colifitness genes during human urinary tract infection. PNAS 111, 18327-18332. doi: 10.1073/ pnas. 1415959112

Subashchandrabose, S., and Mobley, H. L. T. (2015). Virulence and fitness determinants of Uropathogenic Escherichia coli. Microbiol. Spect 3, doi: 10. 1128/micorbiolspec.UTI-0015-2012

Sundén, F., Butler, D., and Wullt, B. (2017). Triggered urine interleukin6 correlates to severity of symptoms in nonfebrile lower urinary tract infections. J. Urol. 198, 107-115. doi: 10.1016/j.juro.2017. 01.070

Tandoğdu, Z., Bartoletti, R., Cai, T., Çek, M., Grabe, M., Kulchavenya, E., et al. (2016). Antimicrobial resistance in urosepsis: outcomes from the multinational, multicenter global prevalence of infections in urology (GPIU) 
study 2003-2013. World J. Urol. 3, 1193-1200. doi: 10.1007/s00345-015$1722-1$

Tandogdu, Z., Cek, M., Wagenlehner, F., Naber, K., Tenke, P., van Ostrum, E., et al. (2014). Resistance patterns of nosocomial urinary tract infections in urology departments: 8-year results of the global prevalence of infections in urology study. World J. Urol. 32, 791-801. doi: 10.1007/s00345-013-1154-8

Thomas, W. E., Nilsson, L. M., Forero, M., Sokurenko, E. V., and Vogel, V. (2004). Shear-dependent 'stick-and-roll' adhesion of type 1 fimbriated Escherichia coli. Mol. Microbiol. 53, 1545-1557. doi: 10.1111/j.1365-2958.2004. 04226.x

Wagenlehner, F. M., Lichtenstern, C., Rolfes, C., Mayer, K., Uhle, F., Weidner, W., et al. (2013). Diagnosis and management for urosepsis. Int. J. Urol. 20, 963-970. doi: 10.1111/iju.12200

Wagenlehner, F. M., Pilatz, A., Naber, K. G., and Weidner, W. (2008). Therapeutic challenges of urosepsis. Eur. J. Clin. Invest 38(Suppl. 2), 45-49. doi: 10.1111/j. 1365-2362.2008.02008.x
Conflict of Interest: The study was co-financed by Coloplast A/S which also assisted with the histological examinations and interpretation of these results. The conclusions of the study were in no way influenced by the commercial interests of Coloplast A/S. LN was employed by company Coloplast A/S.

The remaining authors declare that the research was conducted in the absence of any commercial or financial relationships that could be construed as a potential conflict of interest.

Copyright (c) 2019 Nielsen, Petersen, Sterk, Grønnemose, Palarasah, Nielsen, Kolmos, Andersen and Lund. This is an open-access article distributed under the terms of the Creative Commons Attribution License (CC BY). The use, distribution or reproduction in other forums is permitted, provided the original author(s) and the copyright owner(s) are credited and that the original publication in this journal is cited, in accordance with accepted academic practice. No use, distribution or reproduction is permitted which does not comply with these terms. 